\title{
A STUDY ON THE IMPORTANCE OF OPEN SOURCE SOFTWARE
}

\author{
Perminder Kaur \\ Department of IT \\ Kalinga University \\ Raipur- Chhattisgarh
}

\author{
Rahul Chawda \\ H.O.D Computer Science \\ Kalinga University \\ Raipur- Chhattisgarh
}

\begin{abstract}
This paper provides a general history and description of open source software. A feature of the software world over the past years has been the rise of OSS including using and developing OSS-names like Google, E-bay and face book. Now such software is increasing being used in the library environment.
\end{abstract}

In this paper I am providing useful information about software for educational institution introducing digital library concept.

\section{INTRODUCTION}

Open source software (OSS) is computer software that has its underlying 'source-code' made available under a licens

Open source software (OSS) is computer software that has its underlying 'source-code' made available under a licens

Open Source software is a computer software that has its underlying "Source Code" made under available under a license. Open source software helps us became better programmer because open source code is publicly accessible, students can easily study it as they learn to make better software. It is a computer software where the source code is made publicly available. Items one can modify software according to their needs. User can fix the bugs improve functions etc.

- NIST (National institute of standard and technology)

Open Source Software is COMMERCIAL* software! • "software for which the human-readable source code is available for use, study, reuse, modification, enhancement, and redistribution by the users of that.

Some popular type of open source software available with its source code available for modification:-

- Mozilla Firefox Web Browser
- Thunderbird Email Client

- PHP scripting Language

- Python Programming Language

- Apache HTTP Web Services

One of the best example of open source software

The Linux kernel is a prominent example of free and open source software. It is a Unix-like operating system released under the GNU General Public License version (GPLv2)

The open source initiative (OSI) is the steward of the OPEN SOURCE DEFINATION and part of its function is to review and approve license conforming to the OSD. Many different license satisfy the OSD, but the type of validation they impose can vary quiet widely. The OSD has developed ten criteria to determine whether a license of software is open source.

1. Free redistribution: the software to be available for distribution without payment.

2. Source code: the soft to be distributed with the source or well-publicized access to it.

3. Derived Works- the license must allow modification and derived works and must allow them to be distributes under the same terms as the license of the original software.

4. Integrity of the author's source code: distribution of "patch files" used to recreate derived works to be permitted.

5. No discrimination against person or group- the license must not discriminate any person or group of person.

6. No Discrimination field or endeavour: - it may not restrict the program from being used in business.

7. Distribution of license.

8. License must not specified to a product

9. License must not restrict other software

10. License must be technology neutral. 
Some of the OSS library portal

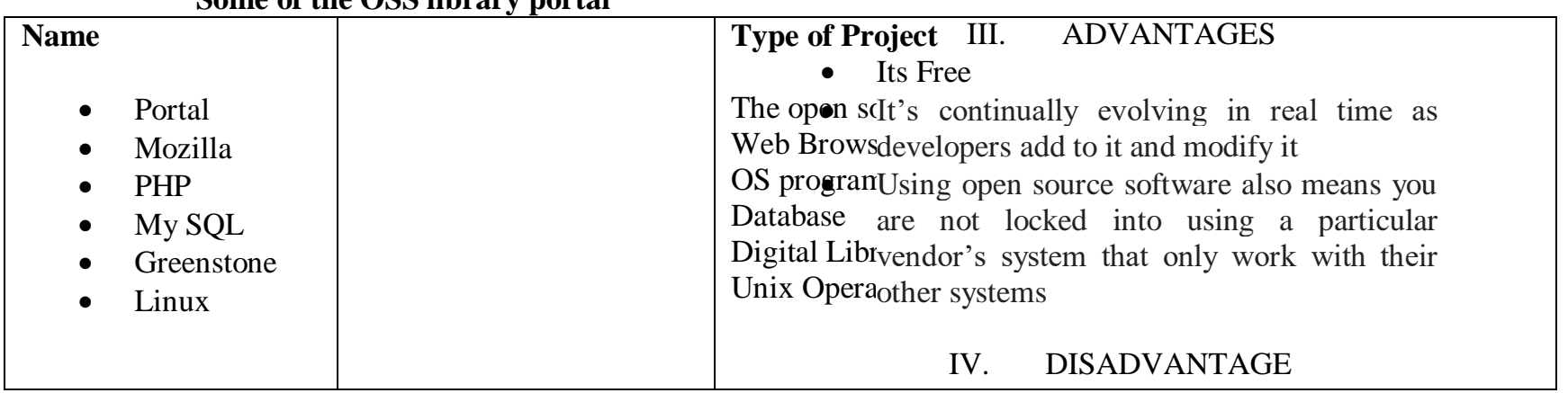

\section{OSS SOFTWARE LICENSE ISSUE}

\section{- OSS and Software Security}

To increase the reliability and security of code, OSS depends directly on the programmer's competence, experience and professional methodology such as peer review, testing, quality audits, alpha and beta versioning etc. However for the library security enforcement, high quality expertise is scarce and may often have to be developed to adequately cope with the increased responsibility that Oss based system will require.

\section{- OSS AND SOFTWARE LICENSE}

There are many different types of OSS license. However it coupled with lack of settled case law and rapidly developing market practice, so library could also consider their procurement operations to make sure they have effective ways of verifying that code they buy or license in does not contain unexpected OSS and other necessary contractual cover.

\section{HOW DOES IT WORK?}

For business user perceptive, open source software works in much same way as proprietary software systems provided by commercial software firms - the only difference being that generally you don't pay for it. However there are a few important differences - the idea behind open source software is that users are effectively codevelopers, suggesting ways to improve it and helping to hunt out bugs and problems.

This means if we wish to modify as per our needs we can port it to another operating system and share it.
- They can be less "user-friendly" and not as easy to use because less attention is paid to developing the user interface

- Although the open source software itself is mostly free, there may still be some indirect costs involved, such as paying for external support.

\section{SO HOW SECURE IS IT?}

A recent report suggests that more than 60 of the Fortune 100 companies may still be using code containing the same vulnerability that led to the Equifax breach. As far as security is concerned, the big win in using open source software is supposed to be transparency. Open source projects mean that everyone and anyone can inspect the source code. At least in theory, the fact that there are "many

Eyes" on the code should mean that bugs and flaws are spotted and fixed quickly.

There's no doubt that open source code is both a boon for businesses and consumers. But it's important to recognize that free code still comes at a cost: the cost of responsibility. It is up to businesses to ensure that their codebase is secure because it is the business that will bear the brunt of any losses, both financial and reputational.

\section{CONCLUSION}

Common platform for sharing ideas and putting them into action by developers or other people who wishes to improve a product or software. We are in the open source revolution where people collaborate to create producing software or technology. Based upon the advantages of open source technology we can finally conclude that open sources deserves the increasingly popularity.

\section{REFERENCE}

[1] Code. Rebel: Linux and the Open Source Revolution.

[2] Hall Jim . 23 years of free doss free Dos, and how people use free dos today recommendation letter volume 1 pp. 1994 
[3] Haverbeke. Marjin browse and nose s. javascript. Edition 5 . recommendation by Rahul Thakur 1025-1027, 1995

[4] Patron , Ury \& vishery cotton. Bennet the Harvard negotiation project ( framework for negoytials which allowed all involved) $1970 \mathrm{~s}$

[5] Addison T.., Vallabh S. 2002. Controlling oss project risk - an emoerial study of methods used by experienced project in Proc .SAICSIT (pp128-140)

[6] Babar ,MA .; Paik Hye young. (2009). Using scrum in Global software Development.

[7] Janamanchi . B , Katsamakas E, Raghupati W and Gao W. The state and profile for open source software projects in health and medical informtics . Int J Med Inform 2009, 78:457-475.
[8] Kemp R. Current developments in Open Source Software. Comput Law Secur Rev 2009; 25:569582

[9] Trainor C. Open source, crowd source: harnessing the power of the people behind our libraries. Program-Electron Lib 2009; 43: 288-298.

[10] Applebee B. The future of Open Source Software. J Res Pract Inf Tech 2003; 35: 227-236.

[11] Sandra P. Flexible and extensible object and repository architecture (Fedora).Lect Notes Comput Sc 1998; 1513: 41-59. 